\title{
From the journals
}

\section{Concurrent use of ACE inhibitors, diuretics and nonsteroidal anti-inflammatory drugs (NSAIDs) is harmful}

The beneficial effect of ACE inhibitors and diuretics in the management of hypertension and heart failure are antagonised by concurrent use of NSAIDs. ACE inhibitors, diuretics and NSAIDs individually or in combination have been responsible in over $50 \%$ of cases of iatrogenic acute renal failure reported to the Australian Adverse Drug Reactions Advisory Committee (ADRAC). Renal failure is also seen with COX 2 inhibitors and angiotensin II receptor antagonists. The fatality rate for ADRAC cases of renal failure with the concurrent use of ACE inhibitors, diuretics and NSAIDs is 10\%. WHO Drug Information 2003; 17: 235. Prescribers should avoid this dangerous combination.

\section{Fluticasone causes adrenal suppression}

Recently there have been several reports worldwide of adrenal insufficiency in adults and children using inhaled corticosteroids. A search of Health Canada database from 1996 to 2002 did not indicate a single case report of suspected adrenal insufficiency associated with budesonide or beclomethasone. However, there were nine reports of adrenal suppression caused by fluticasone. Five of these were in children. In four cases the dose of fluticasone exceeded $1000 \mu \mathrm{g} /$ day. Doctors should be aware that, beyond a certain limit, increase in the dose is not beneficial but only increases adverse effects. WHO Drug Information 2003; 17: 237 The maximum dose of fluticasone recommended for children is $400 \mu \mathrm{g} / \mathrm{day}$.

\section{Cardiovascular risk should be evaluated before prescribing a coxib}

There is an increased risk of cardiovascular and cerebrovascular diseases with rofecoxib and celecoxib. The risk is known to be greater with rofecoxib than with celecoxib, and appears to be dose related. Rofecoxib should not be used in doses exceeding $25 \mathrm{mg} /$ day. WHO Drug Information 2003; 17: 241

\section{Frusemide is not indicated for routine management of hypertension}

Frusemide is a potent diuretic. It is used in the management of acute left ventricular failure, pulmonary oedema, chronic heart failure, oliguria due to renal failure and diuretic resistant oedema. British National Formulary 2003; 46: 66-7. Although frusemide is sometimes used to lower the blood pressure in patients resistant to thiazide diuretics the British National Formulary does not have hypertension as an indication for frusemide. Hence the diuretics recommended for management of hypertension are the thiazides. In Sri Lanka this means hydrochlorothiazide. Doctors should avoid using frusemide in the routine management of hypertension.

\section{Supporting the bereaved sibling following sudden and unexpected death of a child}

Bereaved children are known to suffer considerable psychological and social difficulties throughout childhood. Their grieving process can be eased by giving a simple explanation of the death, telling the truth that can be understood by the child (considering the age and maturity), avoiding terms such as "passed away", "he is sleeping", or "we lost him", as these can confuse children, and including the children in the planning, and processing of mourning rituals. Current Paediatrics 2003; 13: 555-9

\section{Osteoarthritis: is it related to physical activity?}

From a recent study, it is now known that participation in physical activity does not increase the risk of hip and knee osteoarthritis. Among walkers and runners, there is no association between frequency, pace or training mileage with hip and knee osteoarthritis. Old age, previous joint injury, surgery, and high body mass index are known independent risk factors for hip and knee osteoarthritis. Preventive Medicine 2003; 36: 636-44

\section{Glitazones increase the risk of congestive cardiac failure}

There is an association between rosiglitazone and pioglitazone and congestive heart failure (CHF). The American Heart Association and the American Diabetes Association have given the following guidelines on the use of glitazones.

1. Start at low doses in patients with risk factors for CHF and monitor patients closely for weight gain, peripheral oedema and CHF.

2. Use with caution in patients with mild CHF.

3. Avoid their use in patients with moderate to severe CHF. Reactions 2003; 982: 3.

Kusum de Abrew, Senior Lecturer, Department of Pharmacology, Faculty of Medicine, Colombo, Sri Lanka. E-mail: <271002@celltel.Ik> 